\title{
Perfil dos pesquisadores com bolsa de produtividade em pesquisa do CNPq da área de saúde coletiva
}

\author{
A profile of researchers in public health \\ with productivity grants from the Brazilian \\ $\mathrm{N}$ ational Research Council (CNPq)
}

Rita Barradas Barata 1

Moisés Goldbaum 2

\footnotetext{
1 Departamento de Medicina Social, Faculdade de Ciências Médicas, Santa Casa de São Paulo. Rua Dr. Cesário Motta Jr. 61, 6o andar, São Paulo, SP 01221-020, Brasil. chmedsoc@santacasasp.org.br 2 Departamento de Medicina Preventiva, Faculdade de Medicina, Universi dade de São Paulo. Av. Dr. Arnaldo 455, São Paulo, SP 01246-903, Brasil. mgoldbau@usp.br
}

Abstract Based on information provided by the Lattes curriculum, this study analyzes the profile of researchers in public health with productivity grants from the Brazilian National Research Council (CNPq). The analysi s consi dered the researcher's undergraduate and graduate degrees, field of expertise, scientific output, and publications. The article discusses comparisons between different categories of researchers and data provided by the CNPq Research Group Directory. Most of the researchers (70\%) received thei $r$ undergraduate degrees ei ther in health sci ences, mainly medicine, or the humanities (18\%), mainly sociology. $60 \%$ have Master's or PhDs in collective health, but some 20 to $30 \%$ of researchers, depending on the category, have no specific training in this particular field. Most are currently engaged in epidemiology. Their scientific work, as evidenced by bibliographic output, varies from 10.56 research products per year for level 2C PhD researchers to 6.60 products per year for level $1 \mathrm{~A}$ researchers. For complete articles published in periodicals, the figures are 3.56 and 2.87 , respectively. The output is mainly published in class A international periodicals as well as national periodicals, classes A and B. The periodicals with the greatest concentration of articles are Cadernos de Saúde Pública/ Reports in Public Health and Revista de Saúde Pública/Journal of Public Health.

Key words Science \& Technology; Research Personnel; Research Support

Resumo O artigo analisa, com base nas informações do currículo Lattes, o perfil dos pesquisadores com bolsa de produtividade em pesquisa do CNPq na área de Saúde Col etiva. A análiselevou em conta a formação graduada e pós-graduada, área de atuação, produção e divulgação científica. As comparações são feitas entre as classes de pesquisadores e com dados do di retório de grupos de pesquisa. A mai oria dos pesquisadores (70\%) são formados em Ciências da Saúde, principalmente em Medicina, ou em Ciências Humanas (18\%), principalmente Sociologia. Sessenta por cento fizeram mestrado e doutorado em Saúde Coletiva, mas há entre 20 e 30\% de pesquisadores, dependendo da classe, sem formação específica na área. A mai oria atua em Epide miologia. A produção científica, expressa em produtos bibliográficos, varia de 10,56 produtos/ano de obtenção do doutorado para os pesqui sadores $2 \mathrm{C}$ a 6,60 produtos/ano para os pesquisadores 1A. Para artigos compl etos publicados em periódi cos os valores são 3,56 e 2,87, respectivamente. A produção é divulgada principalmente em periódicos A internacional e, A e B nacional. Os periódicos que concentram a publicação são Cadernos de Saúde Pública e Revista de Saúde Pública.

Palavras-chave Ciência \& Tecnologia; Pesquisadores; Apoio a Pesquisa 


\section{Intro dução}

O processo de diferenciação do mundo social, segundo Bourdieu (2001), implica a diferenciação dos modos de conhecimento, identificando nesse processo novos objetos e princípios de compreensão e explicação que dão origem a campos culturais autônomos, dentre os quais, os campos científicos.

No caso dos campos científicos, eles se distinguem dos demais pela imposição de uma forma regulada e organizada de competição, pelos constrangimentos lógicos e experimentais a que estão sujeitas as formas de conhecimento, bem como pela finalidade do conhecimento neles produzidos. O processo de diferenciação que dá origem aos diversos campos científicos implica, portanto, a especificação de um objeto próprio e de agentes com formação específica para nele atuarem.

Enquanto campo científico, a Saúde Coletiva constitui um conjunto de teorias e práticas que se organiza e se diferencia para compreender, explicar e modificar o processo saúde-doença, em seus aspectos materiais e não materiais, na dimensão coletiva, assim como as formas de respostas socialmente organizadas para o enfrentamento das necessidades de saúde (Paim \& Almeida Filho, 2000). Tomando esses aspectos da realidade como objeto de trabalho, no Brasil, a Saúde Coletiva experimentou crescimento considerável na última década, expresso no aumento do número e ampliação da distribuição de programas de pós-graduação, constituição de grupos de pesquisa, número de pesquisadores qualificados e consolidação da produção científica, retratados, por exemplo, em recente levantamento sobre a pesquisa em Epidemiologia no Brasil (Guimarães et al., 2001).

Esse crescimento se expressa também em maior demanda por recursos de financiamento a projetos de investigação e bolsas de produtividade em pesquisa (CA Saúde Coletiva, 2002), bem como nas solicitações de bolsas de pósdoutorado e bolsas "sanduíche", junto à Coordenação de Aperfeiçoamento de Pessoal de Nível Superior (CAPES). Com o crescimento constante da área, a proporção dos bolsistas tende a representar parcela cada vez menor do conjunto de pesquisadores, e a pressão por bolsas de produtividade tende a aumentar levando à adoção de critérios de seleção cada vez mais restritivos. Assim, é importante definir que características interessam ao fortalecimento e à consolidação do campo para que a concessão das bolsas participe, organicamente, dos esforços nesse sentido. Este trabalho tem por objetivo estabelecer o perfil dos pesquisadores com bolsa de produti- vidade em pesquisa na área da Saúde Coletiva do Conselho Nacional de Desenvolvimento Científico e Tecnológico (CNPq), com vistas a identificar as repercussões produzidas com base na aplicação de critérios de julgamento utilizados pelos membros do Comitê Assessor em Saúde Coletiva e Nutrição do CNPq (CA Saúde Coletiva, 2002), além de fornecer informações úteis para o delineamento da política de desenvolvimento científico e tecnológico no campo.

\section{Método}

A lista de bolsistas de produtividade com bolsas ativas em dezembro de 2002, na área da Saúde Coletiva foi obtida no portal do CNPq. Identificados os bolsistas, foram consultados os currículos Lattes de todos os pesquisadores para cada uma das classes, a saber, pesquisadores $2 \mathrm{~A}, 2 \mathrm{~B}, 2 \mathrm{C}$ e $1 \mathrm{~A}, 1 \mathrm{~B}$ e $1 \mathrm{C}$.

Com base nas informações existentes nos currículos Lattes foi construído o banco de dados contendo informações relativas à formação de graduação e pós- graduação, tempo de obtenção do doutorado, produção científica e divulgação da produção em artigos científicos nos últimos três anos. A análise de consistência dessas informações não foi aqui realizada dada a natureza exploratória deste trabal ho.

Os pesquisadores foram classificados em cada nível de formação, segundo as grandes áreas do conhecimento destacando-se, no caso da pós-graduação, a formação específica em Saúde Coletiva. Além da classificação por grandes áreas, levou-se em consideração a realização da formação no país ou no exterior.

A produção científica foi computada baseando-se no quadro resumo existente no currículo Lattes, referindo-se ao período todo de atividade do pesquisador. Para permitir a comparação entre as classes em termos de produtividade, optou-se por utilizar o tempo médio de obtenção do doutorado em cada classe como referência temporal para o ajuste.

Para a caracterização da divul gação científica foram classificados os artigos publicados em periódicos científicos nos últimos três anos (2000 a 2002), para cada um dos pesquisadores bolsistas. Os periódicos foram classificados segundo a proposta adotada pela CAPES, no sistema QUALIS, baseada nas exigências de indexação das bases de dados nacionais e internacionais (CAPES, 2003). Os periódicos foram também classificados em pertencentes ou não à área da Saúde Coletiva com base em seus títulos. Foram considerados como periódicos da área aqueles que, no título continham menção 
à saúde ou a alguma das disciplinas ou áreas temáticas do campo da Saúde Coletiva, tais como, epidemiologia, saúde ocupacional, odontologia preventiva etc. As comparações foram feitas entre as classes e, para algumas variáveis entre as classes e a média do conjunto de pesquisadores.

A data da última atualização para todos os currículos analisados variou de fevereiro a dezembro de 2002 , sendo que $70 \%$ dos currículos haviam sido atualizados entre setembro e dezembro de 2002. Trata-se portanto de informações atualizadas, para a maioria dos pesquisadores.

\section{Distribuição dos pesquisadores bolsistas}

Segundo os dados do Diretório de Grupos de Pesquisa (CNPq, 2002), existem 5.017 grupos $(33,1 \%)$, que referiram a saúde humana como o setor de aplicação dos conhecimentos por eles produzidos. Destes, $388(2,1 \%)$ referiram a área de Saúde Coletiva como a área de conhecimento predominante. Esses grupos registraram 2.115 pesquisadores, sendo 1.129 com titulação de doutorado, dos quais 433 com posição de liderança em seus respectivos grupos.

Na última edição, o diretório introduziu a atribuição de escores padronizados relativos à qualificação, à produção e às orientações concluídas, de forma a poder separar os grupos de pesquisa em grupos consolidados, grupos em consolidação e em formação. Para 344 grupos foi possível estabelecer escores, com base nos quais, os grupos foram classificados. Destes, 100 $(29,07 \%)$ foram classificados como grupos consolidados, 100 (29,07\%) como em consolidação e 144 (41,86\%) como grupos em formação.

Supondo que a cobertura dessa base de dados corresponda a $100 \%$ dos grupos em atividade na área, uma vez que, entre as instituições que não forneceram informações não há nenhuma com atuação na área, podemos estimar a existência de 1.129 doutores com atividades de pesquisa no campo da Saúde Coletiva. Os bolsistas de produtividade do CNPq somam 115 , correspondendo assim a $10,18 \%$ dos pesquisadores em atividade. A sua distribuição segundo classes pode ser visualizada na Tabela 1.

O conjunto dos doutores em atividade é formado por 461 homens (40,83\%) e $668 \mathrm{mu}$ Iheres (59,17\%), apresentando composição semelhante à dos pesquisadores bolsistas. Entretanto, a distribuição por sexo segundo classes (Tabela 2), mostra diferenças significantes (não há informações disponíveis sobre a idade dos pesquisadores). Nas duas classes iniciais, $2 \mathrm{C} \mathrm{e}$ $2 \mathrm{~B}$, há predomínio de mulheres; na classe $2 \mathrm{~A}$ a distribuição é igual para ambos os sexos e a partir da classe 1C passam a predominar os homens. Assim, para as mulheres a distribuição configura uma pirâmi de de base larga e topo afunilado, enquanto que para os homens a figura é invertida com maior concentração na última classe e menor nas duas primeiras.

Os pesquisadores bolsistas pertencem a 26 diferentes instituições, das quais 59\% são universidades federais e $18 \%$ universidades estaduais. As restantes distribuem-se entre institutos de pesquisa, instituições de ensino superior e universidades privadas. Os pesquisadores I estão concentrados em 15 instituições e 21 departamentos, sendo que apenas dez delas reúnem $90 \%$ dos pesquisadores e $50 \%$ pertencem apenas a duas (Fundação Oswaldo Cruz - FIOCRUZ - e Universidade de São Paulo - USP).

Tabela 1

Distribuição dos bolsistas da área de Saúde

Coletiva segundo classe. CNPq, 2002.

\begin{tabular}{lcc}
\hline Classe & $\mathrm{n}$ & $\%$ \\
\hline 1A & 19 & 16,52 \\
1B & 14 & 12,17 \\
1C & 16 & 13,91 \\
2A & 20 & 17,39 \\
2B & 22 & 19,13 \\
2C & 24 & 20,87 \\
Total & 115 & 100,00 \\
\hline
\end{tabular}

Fonte: Plataforma Lattes.

Tabela 2

Distribuição dos pesquisadores por sexo segundo classe. CNPq, 2002.

\begin{tabular}{lrrrr}
\hline Classe & \multicolumn{2}{c}{ Masculino } & \multicolumn{2}{c}{ Feminino } \\
& $n$ & $\%$ & $n$ & $\%$ \\
\hline 1A & 14 & 73,68 & 5 & 26,32 \\
1B & 8 & 57,14 & 6 & 42,86 \\
1C & 10 & 62,50 & 6 & 37,50 \\
2A & 10 & 50,00 & 10 & 50,00 \\
2B & 8 & 36,36 & 14 & 63,64 \\
2C & 6 & 25,00 & 18 & 75,00 \\
Total & 56 & 48,70 & 59 & 51,30 \\
\hline
\end{tabular}

$\mathrm{p}=0,0223$.

Fonte: Plataforma Lattes. 
Esta concentração é semelhante à observada para a área da medicina, se considerarmos como base de cálculo as 88 instituições que mencionam possuir grupos de pesquisa em Saúde Coletiva no diretório do CNPq (Zago et al., 2001). A concentração é menor se considerarmos apenas as 49 instituições que possuem grupos consolidados e/ ou em consolidação, excluindo aquelas que têm apenas grupos em formação.

Os pesquisadores I estão distribuídos em 13 (62\%) departamentos ou centros da área da Saúde Coletiva, 4 (19\%) departamentos ou centros clínicos, 2 (9,5\%) departamentos ou centros de ciências biológicas e 2 (9,5\%) departamentos ou centros de outras áreas do conhecimento, fora das ciências da saúde. Dos pesquisadores I, 36 (73\%) estão vinculados a um dos 27 programas de pós-graduação em Saúde Coletiva credenciados pela CAPES, 12 (24\%) pertencem a programas de outras áreas e 1 (2\%) não está vinculado a programas de pós-graduação. Apenas 12 (44\%) dos 27 programas de pós-graduação têm pesquisadores I em seus quadros.

\section{Características de formação}

Paim \& Almeida Filho (2000:59), afirmam que a Saúde Coletiva pode ser entendida como "campo científico, onde se produzem conhecimentos e saberes acerca do objeto saúde e, onde operam distintas disciplinas que o contemplam sob vários ângulos; e como âmbito de práticas, onde se real izam ações em diferentes organizações e institui ções por diversos agentes, dentro e fora do espaço convencionalmente reconhecido como setor saúde".

Este caráter inerentemente multidisciplinar, politemático e os limites amplos e pouco definidos do campo, trazem ao mesmo algumas características peculiares. A aquisição das disposições específicas exigidas pela prática em um dado campo científico se realiza por meio da formação, isto é, do processo que envolve a relação entre as disposições primárias (domésticas ou cotidianas) e as constrições próprias à estrutura do campo (aquisição do habitus) (Bourdieu, 2001).

Entre os pesquisadores bolsistas este caráter multidisciplinar se expressa pela diversidade da formação de graduação. Conforme o esperado, 77,4\% dos pesquisadores têm sua formação de graduação em Ciências da Saúde seguindo-se a formação em Ciências Humanas para $12,2 \%$. Os restantes $10,4 \%$ distribuem-se entre as Ciências Biológicas, as Ciências Exatas, as Ciências Agrárias e a Lingüística. Ne- nhum dos pesquisadores tem formação de graduação em Engenharias (Tabela 3).

Se a Saúde Coletiva é um campo científico diferenciado e se a socialização dos cientistas exige a aquisição de um habitus específico ao campo, então a formação na área deve ser um dos requisitos para a caracterização dos pesquisadores do campo.

A maioria dos bolsistas (88,7\%) fez mestrado, sendo maior sua proporção entre pesquisadores das classes $2 A, 2 B$ e $2 C(p=0,007)$. Cerca de $60 \%$ dos mestrados foram feitos em Saúde Coletiva, variando de $47,8 \%$ para os pesquisadores $2 \mathrm{C}$ a $72,2 \%$ para os $2 \mathrm{~A}(\mathrm{p}=0,17)$. Parece paradoxal que entre os pesquisadores $2 \mathrm{C}$, formados em média há menos tempo, predomine a formação em outras áreas do conhecimento (Tabela 4).

Entretanto, existem situações nas quais os pesquisadores optam por formação pós-graduada na própria área disciplinar na qual fizeram sua formação de graduação, embora adotem o objeto saúde, na dimensão coletiva, como objeto da sua produção. Assim, a formação pós-graduada, na área específica, não pode ser tomada como critério absoluto para a identificação dos pesquisadores e, portanto, para a concessão de bolsas ou auxílios à pesquisa.

Apenas quatro dos pesquisadores, todos bolsistas da classe $1 \mathrm{~A}$, não possuem doutorado. Dos demais, $60 \%$ possuem doutorado em Saúde Coletiva. Os restantes $40 \%$ têm doutorado em ciências da saúde (exceto Saúde Coletiva), ciências humanas, ciências biológicas, engenharias, ciências exatas e ciências agrárias. A proporção de pesquisadores com doutorado em Saúde Coletiva é semelhante nas seis classes $(p=0,42)$, variando entre $50 \%$ nas classes 1B e $2 \mathrm{~B}$ a $75 \%$ na classe 2A (Tabela 5). Da mesma forma que para o mestrado, a grande proporção de pesquisadores com doutorado em outras áreas que não a Saúde Coletiva reflete a multidisciplinaridade do campo, mas pode também estar indicando a falta de limites claros nessa área do conhecimento. Tendo em vista que o doutorado destina-se explicitamente à preparação de pesquisadores, o fato de parcela importante dos bolsistas de produtividade em pesquisa da Saúde Coletiva não terem formação específica neste campo constitui-se em questão a ser detidamente analisada. Entretanto, foge ao escopo do presente artigo aprofundar essa análise.

Quanto ao local de realização do doutorado observa-se que entre os pesquisadores 2C (96\%) realizaram sua formação no país. Esta proporção diminui progressivamente nas classes subseqüentes chegando a $50 \%$ entre os pesquisado- 
Distribuição dos pesquisadores bolsistas segundo área de conhecimento na formação de graduação e classe. CN Pq, 2002.

\begin{tabular}{|c|c|c|c|c|c|c|c|c|c|c|c|c|}
\hline \multirow[t]{2}{*}{ Área } & \multicolumn{2}{|c|}{$1 \mathrm{~A}$} & \multicolumn{2}{|c|}{$1 \mathrm{~B}$} & \multicolumn{2}{|c|}{$1 C$} & \multicolumn{2}{|c|}{$2 \mathrm{~A}$} & \multicolumn{2}{|c|}{$2 \mathrm{~B}$} & \multicolumn{2}{|c|}{$2 C$} \\
\hline & $\mathrm{n}$ & $\%$ & $\mathrm{n}$ & $\%$ & $\mathrm{n}$ & $\%$ & $\mathrm{n}$ & $\%$ & $\mathrm{n}$ & $\%$ & $\mathrm{n}$ & $\%$ \\
\hline Ciências Exatas & & & 1 & 7,1 & & & 1 & 5,0 & 1 & 4,5 & & \\
\hline Ciências Biológicas & & & 1 & 7,1 & 2 & 12,5 & & & & & 4 & 16,7 \\
\hline Ciências da Saúde & 16 & 84,2 & 8 & 57,3 & 11 & 68,8 & 16 & 80,0 & 18 & 82,0 & 18 & 75,0 \\
\hline Ciências Agrárias & & & & & & & & & 1 & 4,5 & & \\
\hline Ciências Sociais Aplicadas & & & 1 & 7,1 & & & & & & & & \\
\hline Ciências Humanas & 3 & 15,8 & 3 & 21,4 & 2 & 12,5 & 2 & 10,0 & 2 & 9,0 & 2 & 8,3 \\
\hline Ling üística/Letras/Artes & & & & & 1 & 6,2 & & & & & & \\
\hline Total & 19 & 100,0 & 14 & 100,0 & 16 & 100,0 & 20 & 100,0 & 22 & 100,0 & 24 & 100,0 \\
\hline
\end{tabular}

Fonte: Plataforma Lattes.

Tabela 4

Distribuição dos pesquisadores bolsistas, segundo a área de conhecimento de obtenção do mestrado. CN Pq, 2002.

\begin{tabular}{|c|c|c|c|c|c|c|c|c|c|c|c|c|}
\hline \multirow[t]{2}{*}{ Área } & \multicolumn{2}{|c|}{$1 \mathrm{~A}$} & \multicolumn{2}{|c|}{$1 \mathrm{~B}$} & \multicolumn{2}{|c|}{$1 \mathrm{C}$} & \multicolumn{2}{|c|}{$2 \mathrm{~A}$} & \multicolumn{2}{|c|}{$2 \mathrm{~B}$} & \multicolumn{2}{|c|}{$2 C$} \\
\hline & $\mathrm{n}$ & $\%$ & $n$ & $\%$ & $\mathrm{n}$ & $\%$ & $\mathrm{n}$ & $\%$ & $\mathrm{n}$ & $\%$ & $\mathrm{n}$ & $\%$ \\
\hline Ciências Exatas & 1 & 5,3 & 1 & 7,1 & & & 2 & 10,0 & & & & \\
\hline Ciências Biológicas & 1 & 5,3 & 1 & 7,1 & & & 1 & 5,0 & 2 & 9,1 & 3 & 12,5 \\
\hline Engenharias & & & & & & & & & & & 2 & 8,3 \\
\hline Ciências da Saúde & & & 2 & 14,3 & 1 & 6,2 & 1 & 5,0 & 4 & 18,2 & 7 & 29,2 \\
\hline Ciências Agrárias & & & & & & & & & 1 & 4,5 & & \\
\hline Ciências Humanas & 3 & 15,8 & 2 & 14,3 & 3 & 18,8 & 1 & 5,0 & 1 & 4,5 & & \\
\hline Lingüística & & & & & 1 & 6,2 & & & & & & \\
\hline Saúde Coletiva & 7 & 36,8 & 8 & 57,2 & 7 & 43,8 & 13 & 65,0 & 14 & 63,7 & 11 & 45,8 \\
\hline Nenhuma & 6 & 31,6 & & & 4 & 25,0 & 2 & 10,0 & & & 1 & 4,2 \\
\hline Total & 19 & 100,0 & 14 & 100,0 & 16 & 100,0 & 20 & 100,0 & 22 & 100,0 & 24 & 100,0 \\
\hline
\end{tabular}

Fonte: Plataforma Lattes.

res $1 A(p=0,009)$. Certamente estes dados refletem o efeito de coorte de geração. Os pesquisadores com formação mais recente já contavam com oferta expressiva de programas nacionais, diferentemente dos pesquisadores cuja formação se deu em etapa anterior, na fase de instalação dos primeiros programas de pós-graduação.

Apenas 23,4\% dos pesquisadores têm titulação de livre docente. Destes 27 indivíduos, 20 obtiveram a titulação na área de Saúde Coletiva, 4 em outras ciências da saúde e 3 em outras áreas do conhecimento. Todos os títulos foram obtidos, como seria de se esperar, no Brasil. A titulação de livre-docência permanece sendo uma exigência para carreiras acadêmicas nas universidades paulistas bem como nas univer- sidades federais localizadas no Estado de São Paulo. Tal exigência não existe nas outras universidades federais do país. Há ainda, um conjunto de docentes que obtiveram a titulação na década de 70 como sucedâneo ao mestrado e ao doutorado, visto que iniciaram suas carreiras docentes antes da instalação da pós-graduação senso estrito no país.

Cerca de $44,3 \%$ dos pesquisadores fizeram pelo menos um pós-doutorado. Destes, $28,7 \%$ foram feitos na área da Saúde Coletiva, 5,2\% em outras ciências da saúde e 10,4\% em outras áreas do conhecimento. Apenas dois cursos foram feitos no país enquanto 51 foram realizados no exterior, principalmente em universidades norte-americanas ou inglesas. 
Distribuição dos pesquisadores bolsistas segundo a formação de doutorado por classe. CN Pq, 2002.

\begin{tabular}{|c|c|c|c|c|c|c|c|c|c|c|c|c|}
\hline \multirow[t]{2}{*}{ Área } & \multicolumn{2}{|c|}{$1 \mathrm{~A}$} & \multicolumn{2}{|c|}{$1 \mathrm{~B}$} & \multicolumn{2}{|c|}{$1 C$} & \multicolumn{2}{|c|}{$2 \mathrm{~A}$} & \multicolumn{2}{|c|}{$2 \mathrm{~B}$} & \multicolumn{2}{|c|}{$2 \mathrm{C}$} \\
\hline & $n$ & $\%$ & $\mathrm{n}$ & $\%$ & $\mathrm{n}$ & $\%$ & $\mathrm{n}$ & $\%$ & $\mathrm{n}$ & $\%$ & $\mathrm{n}$ & $\%$ \\
\hline Ciências Exatas & & & & & & & 1 & 5,0 & & & & \\
\hline Ciências Biológicas & & & 2 & 14,3 & 1 & 6,2 & 1 & 5,0 & 1 & 4,5 & 2 & 8,3 \\
\hline Engenharias & & & & & & & & & & & 2 & 8,3 \\
\hline Ciências da Saúde & 3 & 15,8 & 4 & 28,6 & 1 & 6,2 & 2 & 10,0 & 7 & 31,9 & 5 & 20,8 \\
\hline Ciências Agrárias & & & & & & & & & 1 & 4,5 & & \\
\hline Ciências Humanas & 2 & 10,5 & 1 & 7,1 & 4 & 25,0 & 1 & 5,0 & 2 & 9,1 & 1 & 4,2 \\
\hline Saúde Coletiva & 9 & 47,4 & 7 & 50,0 & 10 & 62,6 & 15 & 75,0 & 11 & 50,0 & 14 & 58,4 \\
\hline Nenhuma & 4 & 21,1 & & & & & & & & & & \\
\hline Total & 19 & 100,0 & 14 & 100,0 & 16 & 100,0 & 20 & 100,0 & 22 & 100,0 & 24 & 100,0 \\
\hline
\end{tabular}

Fonte: Plataforma Lattes.

Assim, em todos os níveis de formação pós-graduada chama a atenção o número expressivo (20\% em média) de bolsistas sem formação específica na área da Saúde Coletiva, embora estejam incluídos entre os pesquisadores do campo. Esta proporção varia entre $10 \%$ para os pesquisadores $2 \mathrm{~A}$ e $29 \%$ para os pesquisadores 2C. Paradoxalmente, a proporção de indivíduos sem formação específica na área é maior nas duas classes iniciais, 2B e 2C. Dado o crescimento do campo e a demanda crescente por bolsas pelos novos doutores é de se estranhar que, justamente nas classes iniciais, cerca de 1/ 3 dos bolsistas não apresentem formação em Saúde Coletiva. Embora a presença de cerca de $20 \%$ mostre um conjunto apreciável de bolsistas sem formação específica no campo, refletindo sua multidisciplinaridade, isso pode estar a indicar a ausência de limites mais precisos na compreensão do campo por parte dos membros do Comitê e, extensivamente, da própria comunidade. Esta questão, evidentemente, se impõe, pois como salientamos cresce a pressão por bolsas de produtividade, o que leva a reforçar a idéia de que um dos critérios para seleção fosse a formação específica em Saúde Coletiva. De outro lado, levando em consideração que o doutorado tem um forte componente de formação de pesquisadores, este fato pode estar gerando uma massa de docentes/ pesquisadores cuja vocação não se adequa exatamente ao campo, com repercussões importantes para o seu pleno desenvolvimento.

Outra possibilidade seria considerar que a produção científica nesse campo não exige formação específica o que corresponderia, em certa medida, à negação da especificidade dos objetos e métodos de investigação próprios a essa dimensão da vida social.

\section{Áreas de atuação dos pesquisadores}

A informação relativa às áreas de atuação é bastante variada por se tratar de informação sem pré-codificação no currículo Lattes. Assim, foram agrupadas em algumas disciplinas ou áreas para facilitar a comparação entre as classes. Cada pesquisador informou entre uma e seis áreas de atuação. As médias, segundo as classes de pesquisadores, variaram de 1,93 para os pesquisadores $2 \mathrm{C}$ a 2,94 entre os $1 \mathrm{~A}$.

Cerca de $93 \%$ dos pesquisadores mencionaram como área de atuação a Saúde Coletiva ou equivalente (Saúde Pública, M edicina Preventiva, Medicina Social, disciplinas específicas do campo tais como saúde ocupacional, saúde mental, saúde materno-infantil, etc.) Dentre as disciplinas do campo merecem destaque a Epidemiologia, mencionada por $69,6 \%$ dos pesquisadores; as Ciências Sociais em Saúde mencionadas por 18,3\% e a Estatística e a modelagem matemática mencionadas por $14,8 \%$ dos pesquisadores. Do total, 28,7\% referiram ter atuação em áreas clínicas e 8,7\% em ciências biológicas. Pelo menos $42,6 \%$ dos pesquisadores que estão recebendo bolsas de produtividade em Saúde Coletiva mencionam atuar em outras áreas do conhecimento, exclusiva ou de maneira associada à sua atuação em Saúde Coletiva.

Estes dados apontam novamente para a necessidade de maior precisão na delimitação do campo da Saúde Coletiva enquanto área de conhecimento específica, ainda que o setor de aplicação saúde possa e deva comportar con- 
tribuições de todas as áreas do conhecimento. Para tornar mais clara esta distinção, os dados do Diretório de Pesquisas do CNPq, versão 4.0, são esclarecedores. Das 2.253 linhas de pesquisa referidas por 1.250 grupos de pesquisadores, tendo como área de conhecimento a Saúde Coletiva, apenas $63,43 \%$ foram referidas como tendo aplicação em saúde humana. Se a estas acrescentarmos $1,82 \%$, referidas à administração pública e seguridade social, teríamos $65,25 \%$. As demais referem a aplicações que vão desde fabricação de coque, álcool, combustíveis nucleares e refino de petróleo $(0,04 \%)$ até a educação (9,76\%) (Tabela 6).

Considerando que apenas 388 grupos de pesquisa foram inscritos na área da Saúde Coletiva, existem outros 862 que mencionam pelo menos uma linha de pesquisa em Saúde Coletiva identificando, entretanto, o setor de aplicação desses conhecimentos nas mais diversas atividades produtivas, nas ciências básicas e em atividades sociais diferentes da saúde humana.
Aparentemente, tudo aquilo que faz parte do conceito ampliado de saúde, tal como consta da Constituição Brasil eira, está sendo identificado como campo de aplicação de conhecimentos em Saúde Coletiva. O fato de um dado conhecimento vir a ser aplicável, imediata ou mediatamente, no setor saúde, não significa, necessariamente, que sua produção faça parte do campo científico da Saúde Coletiva.

\section{Produção científica}

A análise da produção científica revela diferenças significantes para todos os tipos de produtos entre as classes de pesqui sadores. Há um nítido gradiente na produção bibliográfica média que vai de 68,21 produtos por pesquisador na classe $2 \mathrm{C}$ a 140 produtos por pesquisador na classe 1A (Tabela 7).

O número médio de artigos completos publicados em periódicos científicos apresenta

Tabela 6

Distribuição das linhas de pesquisa em Saúde Coletiva mencionadas por diferentes grupos, segundo setor de aplicação do conhecimento, 2002.

\begin{tabular}{|c|c|c|}
\hline Setor de aplicação & $\mathrm{n}$ & $\%$ \\
\hline Saúde humana & 1.429 & 63,43 \\
\hline Educação & 220 & 9,76 \\
\hline Meio ambiente e desenvolvimento sustentado & 100 & 4,44 \\
\hline Produtos e processos biotecnológicos & 60 & 2,66 \\
\hline Mercado de trabalho e mão-de-obra & 57 & 2,53 \\
\hline Administração pública, defesa e seguridade social & 41 & 1,82 \\
\hline Recreação, cultura, artes e desporto & 36 & 1,60 \\
\hline Desenvolvimento urbano & 31 & 1,38 \\
\hline Manejo industrial de água, dejetos e atividades conexas & 27 & 1,20 \\
\hline Desenvolvimento rural & 23 & 1,02 \\
\hline Agricultura, pecuária, avicultura, exploração florestal & 23 & 1,02 \\
\hline Assessoria e consultoria a empresas & 19 & 0,84 \\
\hline Política, planejamento e gestão habitacional e das cidades & 10 & 0,44 \\
\hline Indústria química & 10 & 0,44 \\
\hline Neurociências & 6 & 0,27 \\
\hline Indústria de software e serviços de informática & 5 & 0,22 \\
\hline Desenvolvimento de novos materiais & 4 & 0,18 \\
\hline Fabricação de equipamentos de instrumentação e automação industrial & 3 & 0,13 \\
\hline Construção civil & 3 & 0,13 \\
\hline Indústria da imprensa, discos, fitas, vídeos e filmes & 2 & 0,09 \\
\hline Estruturas metálicas, caldeiraria pesada, tanques, reservatórios & 2 & 0,09 \\
\hline Previsão do tempo e prospecção climática & 1 & 0,04 \\
\hline Logística de transporte, armazenamento e comunicações & 1 & 0,04 \\
\hline Fabricação de coque, de álcool e de combustíveis nucleares, refino de petróleo & 1 & 0,04 \\
\hline Outros & 139 & 6,17 \\
\hline
\end{tabular}

Fonte: Diretório dos Grupos de Pesquisa, CN Pq. 
Produção média* segundo diferentes produtos e classes dos pesquisadores bolsistas. CN Pq, 2002.

\begin{tabular}{|c|c|c|c|c|c|c|}
\hline Produtos & $1 \mathrm{~A}$ & 1B & $1 \mathrm{C}$ & $2 \mathrm{~A}$ & 2B & $2 \mathrm{C}$ \\
\hline Produção bibliográfica & 140,00 & 121,36 & 125,81 & 110,75 & 92,50 & 68,21 \\
\hline Artigos completos & 60,89 & 50,28 & 45,88 & 31,25 & 28,59 & 23,00 \\
\hline Resumos em periódicos & 4,05 & 2,35 & 7,00 & 8,65 & 6,00 & 3,29 \\
\hline Trabalhos completos em anais & 5,84 & 6,64 & 7,25 & 7,70 & 4,27 & 6,13 \\
\hline Resumos em anais & 41,05 & 46,64 & 46,88 & 49,10 & 40,27 & 28,00 \\
\hline Livros & 3,05 & 2,14 & 0,94 & 2,40 & 1,73 & 0,63 \\
\hline Capítulos de livros & 12,53 & 10,43 & 9,81 & 6,65 & 5,09 & 4,00 \\
\hline Livros organizados & 1,63 & 0,43 & 0,69 & 1,05 & 0,50 & 0,38 \\
\hline O utras produções bibliográficas & 0,95 & 2,50 & 6,88 & 19,10 & 6,18 & 2,75 \\
\hline Produção técnica & 22,84 & 43,79 & 33,50 & 32,40 & 17,55 & 19,46 \\
\hline
\end{tabular}

* Total da produção referida pelo conjunto de pesquisadores dividida pelo número de pesquisadores.

Fonte: Plataforma Lattes.

gradiente semelhante, havendo diferença média de 37,89 artigos por pesquisador entre as classes 1A e 2C. Para os resumos publicados em periódicos não há um padrão definido. Provavelmente, a composição de cada classe quanto às áreas temáticas é diferente e nas diferentes áreas a prática de publicar resumos em periódicos é variada. Quanto aos resumos publicados em anais, as médias são semelhantes entre as quatro classes diferindo apenas na $2 \mathrm{~B}$ e $2 \mathrm{C}$, onde os valores são menores.

A média de livros publicados também é variada sendo comparativamente menor nas classes $1 \mathrm{C}$ e 2C. Neste caso também a composição de cada classe segundo áreas temáticas pode ter influência na escolha deste tipo de produto para divulgação de resultados de pesquisa. Os capítulos de livros apresentam o gradiente esperado entre as classes enquanto os livros organizados variam irregularmente. No caso de outras publicações e da produção técnica há muita variação no preenchimento de cada currículo tornando esses dados menos confiáveis. Entretanto, observa-se um gradiente nítido em direção às classes $2 \mathrm{~A}, 2 \mathrm{~B}$ e $2 \mathrm{C}$ (Tabela 7 ).

Evidentemente, a produção em cada uma das classes consideradas é fortemente influenciada pelo tempo médio de obtenção do doutorado dos pesquisadores que a compõe. As médias e os intervalos de confiança do tempo de obtenção do doutorado estão apresentados na Tabela 8. Embora a variação no interior das classes seja grande, principalmente nas $1 \mathrm{C}$ e $1 \mathrm{~A}$, a diferença entre as médias é significante. Nas três primeiras classes a precisão das estimativas está prejudicada pelo pequeno número de indivíduos e pela variação grande em duas das três classes, mas as estimativas pontuais são diferentes e gradativas. Nas três classes seguintes a precisão é mais satisfatória tanto pelo número maior de indivíduos em cada classe quanto pela maior homogeneidade interna.

A Tabela 9 apresenta os dados de produção corrigidos pelo tempo médio de obtenção do doutorado, permitindo assim a avaliação da produtividade de cada classe de pesquisadores. Os valores em negrito e itálico na Tabela 9 correspondem àqueles que ultrapassaram a média do conjunto de pesquisadores, obtida pela divisão entre a produção média total e o número médio de anos de conclusão do doutorado para o conjunto de pesquisadores. Pode-se observar que, relativamente, a classe mais produtiva é aquela dos pesquisadores $2 \mathrm{~A}$ seguida pelas classes $2 \mathrm{C}$ e $1 \mathrm{C}$. As menos produtivas são justamente as classes $1 \mathrm{~A}$ e $1 B$, uma vez que a primeira destacou-se apenas na produção e organização de livros, mesmo assim apresentando valores inferiores aos de outras classes, e a segunda, ultrapassando o valor médio apenas para a produção técnica ficando, entretanto, abaixo de outras classes.

Estes dados sugerem que as coortes de pesquisadores com formação mais recente apresentam, em média, perfil mais produtivo, ao menos em relação aos tipos de produtos considerados, coincidindo com a maior importância atribuída, mais recentemente, à produção de conhecimentos científicos como atividade inerente ao trabalho acadêmico.

A Tabela 10 compara a produtividade dos bolsistas pesquisadores à produtividade dos doutores em geral e dos doutores em atuação nos grupos de pesquisa consolidados, da gran- 
de área das ciências da saúde. Os pesquisadores bolsistas apresentam produtividade mais alta do que o conjunto de doutores em atividade na grande área da saúde. Para todos os tipos de produtos, exceto trabalhos completos publicados em anais de eventos científicos, a média de produção dos bolsistas é mais alta. Mesmo quando se procede a uma comparação restrita apenas aos doutores que participam dos grupos de pesquisa consolidados, a produção dos bolsistas permanece mais alta.

De acordo com o esperado, os pesquisadores classificados na classe 1 apresentam participação expressivamente maior na formação de novos pesquisadores, principalmente em nível de doutorado. O gradiente pode ser observado tanto para os valores médios quanto para a densidade, isto é, o valor médio corrigido pelo tempo médio de conclusão do doutorado. $\mathrm{O}$ conjunto dos doutores em atividade na área apresenta em média 0,15 orientações de doutorado por ano e 0,42 de mestrado por ano (Tabela 11).

\section{Divulgação da produção científica}

Os pesquisadores bolsistas publicaram 1.124 artigos completos em periódicos científicos no período 2000 a 2002, podendo haver alguma duplicação na contagem em decorrência de co-autorias. Diferentemente do que havia sido observado para a produção ao longo da vida, quando são considerados apenas os artigos completos publicados em periódicos nos últimos três anos, a produtividade é diretamente proporcional à classificação do pesquisador bolsista (Tabela 12). Provavelmente, a maior produtividade no período atual, observada em todas as classes de pesquisadores, esteja refletindo a maior importância conferida à produção e divulgação científicas.

Há relativamente maior proporção de publicações em periódicos A internacionais entre os pesquisadores 1 quando comparados aos pesquisadores 2 , ocorrendo o inverso para as publicações C locais. No total, $68 \%$ dos artigos foram publicados em periódicos com circulação internacional, $24 \%$ em periódicos de circulação nacional e apenas $8 \%$ em publicações com circulação local. Dentre os periódicos internacionais, predomina a publicação naqueles de maior impacto (A), assim como para os periódicos nacionais, embora nesses últimos haja proporção semel hante de publicações em periódicos A e B (Tabela 13).

Tabela 8

Distribuição dos pesquisadores bolsistas segundo

o tempo de conclusão do doutorado por classe. CNPq, 2002.

\begin{tabular}{lcc}
\hline Classe & Tempo médio (anos) & Intervalo de confiança \\
\hline IA & 21,21 & $16,43-25,98$ \\
1B & 20,35 & $19,9-20,76$ \\
1C & 14,62 & $8,97-20,27$ \\
2A & 11,89 & $10,57-13,21$ \\
2B & 8,82 & $7,25-10,39$ \\
2C & 6,46 & $5,86-7,06$ \\
\hline
\end{tabular}

$\mathrm{p}=0,00000$

Fonte: Plataforma Lattes.

Densidade de produção* segundo diferentes produtos e classes dos pesquisadores bolsistas. CN Pq, 2002.

\begin{tabular}{|c|c|c|c|c|c|c|c|}
\hline Produtos & $1 \mathrm{~A}$ & 1B & $1 \mathrm{C}$ & $2 \mathrm{~A}$ & $2 \mathrm{~B}$ & $2 \mathrm{C}$ & Valor médio \\
\hline Produção bibliográfica & 6,60 & 5,96 & 8,60 & 9,31 & 10,49 & 10,56 & 8,19 \\
\hline Artigos completos & 2,87 & 2,47 & 3,14 & 2,63 & 3,24 & 3,56 & 2,98 \\
\hline Resumos em periódicos & 0,19 & 0,12 & 0,48 & 0,73 & 0,68 & 0,51 & 0,40 \\
\hline Trabalhos completos em anais & 0,28 & 0,33 & 0,50 & 0,65 & 0,48 & 0,95 & 0,48 \\
\hline Resumos em anais & 1,94 & 2,29 & 3,20 & 4,13 & 4,57 & 4,33 & 3,16 \\
\hline Livros & 0,14 & 0,11 & 0,06 & 0,20 & 0,20 & 0,10 & 0,13 \\
\hline Capítulos de livros & 0,59 & 0,51 & 0,67 & 0,56 & 0,58 & 0,62 & 0,59 \\
\hline Livros organizados & 0,08 & 0,02 & 0,05 & 0,09 & 0,06 & 0,06 & 0,06 \\
\hline O utras produções bibliog ráficas & 0,04 & 0,12 & 0,47 & 1,61 & 0,70 & 0,43 & 0,50 \\
\hline Produção técnica & 1,08 & 2,15 & 2,29 & 2,72 & 1,99 & 3,01 & 2,06 \\
\hline
\end{tabular}

* Média por pesquisador dividida pelo tempo médio de obtenção do doutorado.

Fonte: Plataforma Lattes. 
Comparação entre o valor médio da produção por pesquisador/ano, entre os pesquisadores bolsistas e os pesquisadores doutores da grande área da saúde. CNPq, 2002.

\begin{tabular}{lccc}
\hline Produtos & Bolsistas & Doutores & $\begin{array}{c}\text { Doutores } \\
\text { Grupos consolidados }\end{array}$ \\
\hline Produção bibliográfica & & 3,14 & 3,93 \\
Artigos completos & 8,19 & 1,97 & 2,54 \\
Trabalhos completos em anais & 2,98 & 0,58 & 0,69 \\
Livros & 0,48 & 0,07 & 0,08 \\
Capítulos de livros & 0,13 & 0,49 & 0,59 \\
Livros organizados & 0,59 & 0,03 & 0,03 \\
Produção técnica & 0,06 & 1,41 & $\ldots$
\end{tabular}

Fonte: Plataforma Lattes; Diretório dos Grupos de Pesquisa, CN Pq.

Tabela 11

O rientações concluídas segundo tipo e classe de pesquisador. CN Pq, 2002.

\begin{tabular}{lrcrc}
\hline Classe & Média & Mestrado & & Doutorado \\
& & Densidade & Média & 0,38 \\
IA & 11,00 & 0,52 & 6,05 & 0,33 \\
1B & 13,00 & 0,64 & 6,64 & 0,23 \\
1C & 6,94 & 0,47 & 3,38 & 0,18 \\
2A & 6,70 & 0,56 & 2,15 & 0,15 \\
2B & 6,77 & 0,77 & 1,36 & 0,11 \\
2C & 4,04 & 0,63 & 0,71 & 0 \\
\hline
\end{tabular}

Fonte: Plataforma Lattes.

Esta produção foi divulgada em 321 periódicos, dos quais $96(29,9 \%)$ podem ser considerados da área de Saúde Coletiva, enquanto os demais (225) são de outras áreas de conhecimento. Dos 1.124 artigos publicados, 620 $(55,2 \%)$ foram divulgados em periódicos da área da Saúde Coletiva e 504 (44,8\%) em periódicos de outras áreas do conhecimento. Embora haja grande diversidade de veículos utilizados para a divulgação dos conhecimentos produzidos, os artigos tendem a concentrar-se nos periódicos da própria área, com média de 6,46 artigos por periódico, enquanto nas revistas de outras áreas a média foi de 2,24 artigos por periódico.

Cerca de 31 periódicos concentram 50\% dos artigos. Para todas as classes de pesquisadores, o periódico mais utilizado, concentrando $18 \%$ das publicações foi Cadernos de Saúde Pública. Em seguida aparece a Revista de Saúde Pública, com $8,5 \%$ dos artigos.
A Tabela 14 apresenta a distribuição de artigos em periódicos que foram selecionados por serem os cinco mais utilizados como veículos de divulgação por, pelo menos, uma das classes de pesquisadores. Cadernos de Saúde Pública e Revista de Saúde Pública aparecem com destaque em todas as classes de pesquisadores bolsistas. A Revista Brasileira de Epidemiologia, embora tenha sido criada há apenas cinco anos, já aparece entre os periódicos que concentram maior número de artigos.

Dos 12 periódicos que concentram $43 \%$ dos artigos, 7 são da área da Saúde Coletiva. Outros 20 periódicos, cada um deles concentrando de 3 a 5 artigos, aparecem entre os dez mais utilizados para uma das classes de pesquisadores bolsistas. As diferenças na distribuição dos periódicos entre as classes são estatisticamente significantes. Em parte, elas refletem as preferências temáticas dos pesquisadores bolsistas. 
Número médio de publicações segundo a classificação dos periódicos e a classe dos pesquisadores bolsistas. CNPq, $2000-2002$.

\begin{tabular}{|c|c|c|c|c|c|c|}
\hline Periódico & $1 \mathrm{~A}$ & $1 B$ & $1 \mathrm{C}$ & $2 \mathrm{~A}$ & $2 B$ & $2 \mathrm{C}$ \\
\hline A Internacional & 6,37 & 6,57 & 6,50 & 4,30 & 4,41 & 3,71 \\
\hline B Internacional & 0,58 & 0,57 & 0,25 & 0,85 & 0,64 & 0,79 \\
\hline C Internacional & 1,53 & 0,79 & 0,50 & 0,65 & 0,73 & 0,83 \\
\hline A Nacional & 1,47 & 1,14 & 0,63 & 0,90 & 0,86 & 0,92 \\
\hline B Nacional & 1,26 & 1,43 & 0,75 & 0,60 & 1,00 & 0,50 \\
\hline C Nacional & 0,63 & 0,64 & 0,81 & 0,50 & 0,41 & 0,13 \\
\hline A Local & 0,47 & 0,07 & 0,50 & 0,40 & 0,09 & 0,04 \\
\hline C Local & 0,37 & 0,43 & 0,56 & 0,60 & 0,82 & 0,54 \\
\hline Total & 12,68 & 11,64 & 10,50 & 8,80 & 8,95 & 7,46 \\
\hline
\end{tabular}

Fonte: Plataforma Lattes.

Distribuição dos artigos publicados segundo classificação dos periódicos e classe dos pesquisadores. CNPq, 2000 a 2002.

\begin{tabular}{|c|c|c|c|c|c|c|c|c|c|c|c|c|}
\hline \multirow[t]{2}{*}{ Periódico } & \multicolumn{2}{|c|}{$1 \mathrm{~A}$} & \multicolumn{2}{|c|}{ 1B } & \multicolumn{2}{|c|}{$1 C$} & \multicolumn{2}{|c|}{$2 \mathrm{~A}$} & \multicolumn{2}{|c|}{$2 \mathrm{~B}$} & \multicolumn{2}{|c|}{$2 \mathrm{C}$} \\
\hline & $n$ & $\%$ & $n$ & $\%$ & $\mathrm{n}$ & $\%$ & $n$ & $\%$ & $\mathrm{n}$ & $\%$ & $n$ & $\%$ \\
\hline A Internacional & 121 & 50,2 & 92 & 56,4 & 104 & 61,9 & 86 & 48,8 & 97 & 49,2 & 89 & 49,7 \\
\hline B Internacional & 11 & 4,6 & 8 & 4,9 & 4 & 2,4 & 17 & 9,7 & 14 & 7,1 & 19 & 10,6 \\
\hline C Internacional & 29 & 12,0 & 11 & 6,8 & 8 & 4,8 & 13 & 7,4 & 16 & 8,1 & 20 & 11,2 \\
\hline A Nacional & 28 & 11,6 & 16 & 9,8 & 10 & 6,0 & 18 & 10,2 & 19 & 9,6 & 22 & 12,3 \\
\hline B Nacional & 24 & 10,0 & 20 & 12,3 & 12 & 7,1 & 12 & 6,8 & 22 & 11,2 & 12 & 6,7 \\
\hline C Nacional & 12 & 5,0 & 9 & 5,5 & 13 & 7,7 & 10 & 5,7 & 9 & 4,6 & 3 & 1,7 \\
\hline A Local & 9 & 3,7 & 1 & 0,6 & 8 & 4,8 & 8 & 4,6 & 2 & 1,0 & 1 & 0,6 \\
\hline C Local & 7 & 2,9 & 6 & 3,7 & 9 & 5,3 & 12 & 6,8 & 18 & 9,2 & 13 & 7,2 \\
\hline Total & 241 & 100,0 & 163 & 100,0 & 168 & 100,0 & 176 & 100,0 & 197 & 100,0 & 179 & 100,0 \\
\hline
\end{tabular}

$\mathrm{p}=0,0122$.

Fonte: Plataforma Lattes.

Em síntese, a produção desse grupo de pesquisadores alcança, portanto, divulgação internacional, por meio inclusive de revistas nacionais indexadas, sem descuidar do debate nacional também veiculado em periódicos de boa qualidade, com corpo editorial, periodicidade e acesso eletrônico em alguns casos.

\section{Perfil dos pesquisadores bolsistas \\ Pesquisador $1 \mathrm{~A}$}

O pesquisador $1 \mathrm{~A}$ corresponde a um profissional do sexo masculino, com formação de graduação em Ciências da Saúde, geralmente em Medicina, sem mestrado ou com mestrado em
Saúde Coletiva, com doutorado em Saúde Coletiva, obtido há cerca de 21 anos e atuação na área de Saúde Coletiva, especialmente em Epidemiologia. Sua produção atual (últimos três anos) compreende 13 artigos, dos quais 8,5 publicados em periódicos de circulação internacional (6 classificados como A) e 3,4 em periódicos de circulação nacional. A maioria de suas publicações é veiculada por Cadernos de Saúde Pública, Revista de Saúde Pública e Revista da Sociedade Brasilei ra de Medicina Tropical.

\section{Pesquisador $1 \mathrm{~B}$}

O pesquisador 1B é também do sexo masculino com formação de graduação em Ciências da Saúde, com mestrado e doutorado em Saúde 
Distribuição dos artigos segundo periódicos e classe de pesquisadores. CNPq, 2000-2002.

\begin{tabular}{|c|c|c|c|c|c|c|c|}
\hline Periódicos & $1 \mathrm{~A}$ & 1B & $1 \mathrm{C}$ & $2 \mathrm{~A}$ & 2B & $2 \mathrm{C}$ & Total \\
\hline Cadernos de Saúde Pública (AI) & $41\left(1^{\circ}\right)$ & $34(19)$ & $41(1 \stackrel{0}{)})$ & $28(1 \stackrel{0}{)})$ & $29(10)$ & $29(1 \stackrel{0}{ })$ & 202 \\
\hline Revista de Saúde Pública (AI) & $11(3 \circ)$ & $11\left(2^{\circ}\right)$ & $14\left(2^{\circ}\right)$ & $24\left(2^{\circ}\right)$ & $26\left(2^{\circ}\right)$ & $9(3)$ & 95 \\
\hline $\begin{array}{l}\text { Revista da Sociedade Brasileira } \\
\text { de Medicina Tropical (AN) }\end{array}$ & $12\left(2^{\circ}\right)$ & $4(4 \stackrel{\circ}{)}$ & $3\left(5^{\circ}\right)$ & 0 & $6(4 \stackrel{\circ}{)}$ & $10\left(2^{\circ}\right)$ & 35 \\
\hline Memórias do Instituto O swaldo Cruz (BI) & $6(5)$ & $3(5 ㅇ)$ & $3(5 \div)$ & 0 & $6(4 \stackrel{\circ}{)}$ & $10\left(2^{\circ}\right)$ & 28 \\
\hline Arquivos Brasileiros de Cardiologia (AN) & $6(5)$ & $4(4 \stackrel{\circ}{)})$ & $3(5)$ & $5(3 \circ)$ & 0 & $6(5 \circ)$ & 24 \\
\hline Revista Panamericana Salud Pública (AI) & 4 & 0 & $8\left(3^{\circ}\right)$ & $4(4 \stackrel{\circ}{)}$ & 0 & $7(4 \stackrel{\circ}{)}$ & 23 \\
\hline São Paulo Medical J ournal (CI) & 0 & $3(5 \stackrel{0}{0})$ & 0 & 0 & $7(3 \stackrel{0}{)}$ & $9(3 \circ)$ & 19 \\
\hline Ciência \& Saúde Coletiva (NA) & $8(4 \stackrel{\circ}{)}$ & $3(5 \div)$ & 0 & $3(5 \div)$ & 0 & 0 & 14 \\
\hline Informe Epidemiológico do SUS (BN) & 4 & $9(3 \circ)$ & 0 & 0 & 0 & 0 & 13 \\
\hline Revista Brasileira de Epidemiologia (BN) & 4 & 0 & 0 & $4(4 \stackrel{\circ}{)})$ & $4(5)$ & 0 & 12 \\
\hline Revista Brasileira de Cancerologia (BN) & 5 & 0 & $6(4 \div)$ & 0 & 0 & 0 & 11 \\
\hline International J ournal of Epidemiology (AI) & 4 & $3(5 \div)$ & $3(50)$ & 0 & 0 & 0 & 10 \\
\hline
\end{tabular}

Classificação dos periódicos: $\mathrm{Al}=\mathrm{A}$ Internacional, $\mathrm{AN}=\mathrm{A}$ Nacional, $\mathrm{BI}=\mathrm{B}$ Internacional, $\mathrm{Cl}=\mathrm{C}$ Internacional, $\mathrm{BN}=\mathrm{B} \mathrm{N}$ acional.

Fonte: Plataforma Lattes.

Coletiva, obtido há cerca de vinte anos e atuação na área de Saúde Coletiva, especialmente em Epidemiologia. A produção atual, em termos de artigos científicos é de 11 artigos, dos quais oito publicados em periódicos de circulação internacional (6,5 nível A) 3 em periódicos de circulação nacional. Sua produção é veiculada nos Cadernos de Saúde Pública, Revista de Saúde Pública e Informe Epidemiológico do SUS.

\section{Pesquisador $1 \mathrm{C}$}

As características do pesquisador $1 C$ são: sexo masculino, formação de graduação em Ciências da Saúde, principalmente Medicina, sem mestrado ou com mestrado em Saúde Coletiva, com doutorado em Saúde Coletiva ou Ciências Sociais, obtido há 14 anos, com pósdoutorado em Saú de Coletiva ou Ciências Sociais e atuação na área de Saúde Coletiva, especialmente em Epidemiologia ou Ciências Sociais em Saúde. A produção atual corresponde a dez artigos, dos quais sete publicados em periódicos de circulação internacional $(6,5$ nível A) e dois em periódicos de circulação nacional. A veiculação dessa produção se faz por intermédio dos Cadernos de Saúde Pública, Revista de Saúde Pública e Revista Panamericana de Salud Pública.

\section{Pesquisador 2A}

O pesquisador 2 A pode ser um homem ou uma mulher, com formação de graduação em
Ciências da Saúde, majoritariamente em Medicina, com mestrado e doutorado em Saúde Coletiva, obtido há 12 anos. Tem como área de atuação a Saúde Coletiva, especialmente a Epidemiologia. A produção atual é de nove artigos em três anos, dos quais seis publicados em periódicos de circulação internacional (4 nível A) e 1,5 em periódicos de circulação nacional. Esses artigos são publicados principalmente nos Cadernos de Saúde Pública e na Revista de SaúdePública.

\section{Pesquisador 2B}

O perfil do pesquisador 2B corresponde a uma mulher com formação de graduação em Ciências da Saúde, principalmente em Medicina, mestrado e doutorado em Saúde Coletiva ou outras áreas de Ciências da Saúde. A área de atuação principal é a Saúde Coletiva, especialmente na Epidemiologia. Este pesquisador concluiu o doutorado há nove anos. A produção nos últimos três anos foi de nove artigos dos quais seis publicados em periódicos com circulação internacional (4 nível A) e dois em periódicos de circulação nacional. As revistas nas quais esta produção é divulgada são Cadernos de Saúde Pública e Revista de SaúdePública.

\section{Pesquisad or $2 \mathrm{C}$}

Finalmente, o pesquisador $2 \mathrm{C}$ é uma mulher com formação de graduação em Ciências da Saúde, geralmente Medicina, com mestrado e 
doutorado em Saúde Coletiva ou em outras áreas das Ciências da Saúde, obtido há seis anos. Sua atuação se faz na área da Saúde Coletiva, especialmente na Epidemiologia. Nos últimos três anos publicou sete artigos, sendo cinco em periódicos de circulação internacional (3 nível A) e 1,5 em periódicos de circulação nacional. As revistas que utiliza para divulgar essa produção são Cadernos de Saúde Pública, Revista da Soci edade Brasileira de Medicina Tropical e Memórias do Instituto Oswaldo Cruz.

\section{Conclusões}

Antes das conclusões propriamente ditas, deve-se assinalar alguns problemas metodológicos referentes às informações colhidas. Assim, o principal viés diz respeito à qualidade das informações disponíveis no currículo Lattes e a diferenças no preenchimento do conjunto de informações por parte dos pesquisadores individualmente. Entretanto, como o currículo Lattes é um dos elementos utilizados no julgamento das solicitações das bolsas, consideramos que seria uma fonte adequada para a caracterização do perfil dos bolsistas.

Outro problema a ser levado em conta é a superestimação relativa da produção científica visto que, em alguns casos os mesmos produtos têm mais de um autor e, dois ou mais autores podem ser bolsistas. Nestes casos, a situação mais freqüente é de estarem classificados em diferentes classes, o que atenuaria o efeito da dupla ou tripla contagem no interior da mesma classe, embora o mesmo produto possa estar contado duas vezes no conjunto da produção.

Há entre os pesquisadores bolsistas um forte predomínio de profissionais com formação de graduação em Medicina, refletindo ainda a origem da Saúde Coletiva brasileira, estreitamente vinculada às escolas médicas.

Dentre as disciplinas que compõem o campo da Saúde Coletiva há nítida concentração entre os pesquisadores bolsistas de praticantes da Epidemiologia, e uma presença discreta de outras disciplinas. Este é um aspecto que merece maior atenção, visto que, para a área é essencial a manutenção de seu caráter multidisciplinar. Segundo Paim \& Almeida Filho (2000), a Saúde Coletiva é um campo de conhecimentos de natureza interdisciplinar cujas disciplinas básicas são a epidemiologia, o planejamento/administração de saúde e as ciências sociais em saúde. Assim, é desejável que exista maior equilíbrio interno entre estas disciplinas, na produção de conhecimentos para a área. As bolsas de produtividade entretanto, estão concentradas quase que exclusivamente naqueles atuantes na área de epidemiologia. Tal concentração parece refletir a dificuldade que as agências de fomento têm em atuar em áreas interdisciplinares, cujo escopo científico inclui diferentes modalidades de investigação. O equilíbrio deve ser deliberadamente buscado pelos membros da própria comunidade científica da Saúde Coletiva, buscando evitar as fraturas e o afastamento entre as disciplinas no interior do campo.

Ao lado do desequilíbrio entre as disciplinas que compõem o campo, merece atenção a existência de pesquisadores sem formação específica em Saúde Coletiva e com produção concentrada em outras áreas de atuação.

O fato de parte considerável dos bolsistas não ter formação pós-graduada em Saúde Coletiva, denota imprecisão dos limites do campo e indica a necessidade de discussão e posicionamento da comunidade científica dessa área do conhecimento. Aparentemente, tem havido alguma confusão entre o setor de aplicação dos conhecimentos produzidos e o campo de produção dos conhecimentos, fato que explica, em parte, esta situação.

Este mesmo aspecto se reflete na divulgação da produção científica, uma vez que parte considerável dos artigos são publicados em revistas que não são da área. Se por um lado, este dado mostraria a disposição de fortalecer o diálogo entre a Saúde Coletiva e áreas afins, por outro, pode estar sinalizando a heterogeneidade dessa produção que ao lado de pesquisas em Saúde Coletiva, abriga pesquisas em ciências biológicas, clínica e outras áreas. Evidentemente, para uma idéia mais clara acerca da produção seria necessário analisar o conteúdo desses artigos de modo a poder classificá-los mais corretamente.

A produção científica desse grupo de pesquisadores é quantitativa e qualitativamente relevante, mostrando tendência à maior produtividade entre os pesquisadores que concluíram o doutorado mais recentemente.

Para melhor qualificar o perfil desses pesquisadores seria interessante comparar esses dados com informações equivalentes para pesquisadores de outras áreas científicas na grande área da saúde e, também para pesquisadores de outras áreas do conhecimento, seja em áreas de ciência fundamental, seja em áreas de ciência aplicada ou estratégica. 


\section{Referências}

BOU RDIEU, P., 2001. Meditações Pascalianas. Rio de Janeiro. Bertrand Brasil.

CA SAÚdE COLETIVA (Comitê Assessor em Saúde Coletiva e Nutrição do (N Pq), 2002. Comentários do Comitê Assessor em Saúde Col etiva e Nutrição do CNPq sobre o Julgamento CA06/2002. Rio de Janeiro: Conselho Nacional de Desenvolvimento Científico e Tecnológico. (mimeo.)

CAPES (Coordenação de Aperfeiçoamento de Pessoal de Nível Superior). 2003. Critérios da GrandeÁrea da Saúde. 8 Abril 2003 \ttp:// www.capes.gov. br>.

CNPq (Conselho Nacional de Desenvolvimento Científico e Tecnológico), 2002. Diretório dos Grupos dePesquisa. \tttp://www.cnpq.br>.
GUIMARÃES, R.; LOURENÇO, R. \& COSAC, S. A., 2001. Pesquisa em Epidemiologia no Brasil. Revista de Saúde Pública, 35:321-340.

PAIM, J. S. \& ALMEIDA FILHO, N., 2000. A Crise da Saúde Pública. Salvador. Casa da Qualidade Editora.

ZAGO, M. A.; CARVALHEIRO, J. R.; LUZ, P. L.; MARI, J. J. \& SILVA, L. J., 2001. Ciência no Brasil: Medicina. Rio de Janeiro: Academia Brasileira de Ciências. (mimeo.)

Recebido em 31 de julho de 2003

Versão final reapresentada em 28 de agosto de 2003

Aprovado em 4 de setembro de 2003 